Psychological Medicine, 1983, 13, 483-486

Printed in Great Britain

\title{
EDITORIAL
}

\section{Life events and psychological morbidity: the evidence from prospective studies $^{1}$}

The role of life event stress in the onset of depressive disorders still remains somewhat controversial (Tennant $e t$ al. 1981 a). A recent review of 8 controlled retrospective studies (Lloyd, 1980) found that 3 did not find any significant association between life events and depression. The methodological problems of retrospective studies and the possibility of spuriously positive associations have been discussed in some detail elsewhere (Tennant et al. 1981a). In essence they are, first, the problems of inaccurately dating events and illness to ensure that events were antecedent. This is a particular problem with depressive illness which may be insidious in onset. Secondly, bias of "effort after meaning' may occur in which depressed subjects search their past experience for some explanation for their distress. Cassidy et al. (1957), for example, found that while $50 \%$ of depressed subjects identified a particular stressor as a cause of their illness after clinical consideration, in only $9 \%$ was the stressor considered a likely cause. Prospective studies in which life event experience is assessed prior to the onset of illness can eliminate these problems, and so provide potentially more powerful evidence for a causal association between life event stress and psychological illness, particularly for commoner syndromes such as the neuroses (Paykel, 1978; Tennant et al. 1981 a). This review focuses on the 8 published controlled prospective studies of life events and neuroses or depression.

One potential limitation of some prospective studies is that the time between the initial assessment of life events and follow-up, when mental state is assessed, is unduly long. Although some have argued that subjects are vulnerable to depression for some considerable time after a stressful event (Brown et al. 1973), most clinicians would believe that the depressive response is provoked most commonly in the 'immediate' aftermath of the event. If Brown's proposition is correct, then those prospective studies with a 'delayed' follow-up period (of 6 months or more) should have positive findings. There are 5 such studies, and only one has produced any positive findings. In the first of these, Hornstra \& Klassen (1977) studied two small community samples. In neither instance did subjects who were initially free from depression $(N=131 ; N=265)$ suffer from morbidity (assessed by self-report measures) 12 months or longer after experiencing stressful events. In the second of these studies, Eaton (1978) reanalysed the 2-year follow-up of Myers et al.'s (1972) community subjects, and found a negative correlation $(r=-0 \cdot 16)$ between life events and subsequent psychological disorder. The authors attempted to explain this unexpected finding, suggesting that subjects not accustomed to stressful events (those with initially low event scores) are more susceptible to the impact of subsequent events occurring during the follow-up period. They do not support this speculation with any data. An equally plausible explanation, consistent with findings by Cooper \& Sylph (1973), is that subjects with especially low levels of life stress experience are at a greater risk of depression, possibly due to inadequate social stimulation. In the third of these 'delayed' studies, Andrews (1981) included an 8-month follow-up and found that there was no significant correlation $(r=0.06)$ between antecedent life events and psychological symptoms assessed by the General Health Questionnaire (GHQ). In the fourth of these studies Murphy (1983) used Brown's more sensitive contextual measure of the threat of life events (Brown \& Harris, 1978) in a sample of elderly depressed patients. Life events assessed at the initial admission did not predict relapse at 12-month follow-up. The last of these five 'delayed' prospective studies (Theorell et al. 1975) revealed some positive findings; these are worthy of some detailed examination. In their 12-month follow-up of some 3000 construction workers, the Social Readjustment Rating Scale (SRE) (Holmes \& Rahe,

\footnotetext{
1 Address for correspondence: Professor C. Tennant, Department of Psychiatry, University of Sydney, Concord Hospital, Concord 2139 , Australia.
} 
1967) was used to assess life events, while 'neurosis' was defined as psychological illness requiring 30 or more consecutive days off work. The relative risk of 'neurosis' in the 'high stress' group was 1.5 and was statistically significant. The limitations of the SRE have been discussed elsewhere (Brown et al. 1973; Brown \& Harris, 1978; Tennant et al. 1981 a; Paykel, 1978). This study highlights some of these inadequacies. Of the 23 events with a prevalence greater than $5 \%$ in this sample, most were more often reported by 'neurotics' than by controls. Of these 23 events, some 17 were clearly not independent of illness. Of the 6 events which were clearly independent, only one ("close friend seriously ill') was more common in the neurotic sample. Given that the criterion for 'neurosis' was rather unusual ( $>30$ days off work due to psychiatric illness), it seems that many such subjects may have had chronic psychiatric illness. A combination of this with the inclusion of many 'illness related' life events in the events inventory may have led to erroneous causal interpretations. A plausible explanation for Theorell et al.'s findings is that those subjects with some neurotic impairment at the initial interview generated more life events and were still found to be neurotic at follow-up. The findings may have been quite different had (i) a more sensitive index of neurosis been used (reflecting less chronicity), and (ii) an events measure been used which excluded life events which were likely to be caused by neurosis. In summary, the findings from these 5 'delayed' prospective studies do not readily support the thesis that there is a prolonged 'at risk' period for depression following stressful experience.

Most clinicians would agree, however, that if stressful experiences cause psychiatric morbidity symptoms are most likely to occur in the 'immediate' aftermath of the stressor. There are 4 studies which incorporated briefer follow-up periods ranging from weekly (Grant et al. 1982) to 4 months (Andrews, 1981). In the first of these (Hall et al. 1977), patients with bipolar manic-depressive illness attending a lithium clinic were studied; no significant relation between antecedent life events (assessed on average at monthly visits) and subsequent depressive episodes was found. In one analysis, patients served as their own controls; the frequency of life events reported in the euthymic visit prior to the recurrence of disorder was similar to other euthymic visits. The type of events reported for the 'pre-recurrence' visit was also similar to those reported at other visits. In another analysis, patients who had recurrent illness were compared with matched subjects who remained well. Those who became ill experienced more 'employment' related events than those remaining well. Somewhat surprisingly, this excess was not found in the depressed subjects but in those who became hypomanic.

In the second of these 'brief' prospective studies Grant et al. (1982) followed a mixed sample of psychiatric patients and normal subjects over 3 years; during that time life events (SRE) and psychological symptoms were assessed every 2 months. Four different temporal patterns of relationships between events and symptoms were studied by Fourier analysis. The most important of these, and the one in which a causal association between events and illness might be implicated, was termed 'coherent but one of phase'; event scores and symptom scores both fluctuated between high and low but did so alternatively from one cycle to the next. This pattern was found in only $9 \%$ of the subjects. The authors argued that it was impossible to decide whether events 'caused' symptoms or vice versa. When undesirable events were examined separately in similar analyses only some $8 \%$ of subjects exhibited this pattern. A causal association could not be implicated. The possibility remained that a 2 -month time frame was too long and that event and symptom relationships might be obscured. Thus a subsample was assessed at weekly intervals, but similar negative findings emerged.

The final 2 'brief' prospective studies (Andrews, 1981; Henderson et al. 1982) have incorporated additional methodological safeguards. Andrews (1981) included in his study of general practice attenders only those who, at an earlier interview, had been free of psychological morbidity. This reduced the possibility that any association between life events at first interview and symptoms at second interview was not causal but was a reflection of chronic neurotics generating life events. Furthermore, a path analysis similar to that used by Eaton (1978) was performed to determine the path by which antecedent events might cause symptoms. The coefficient for the causal path between life events and neurosis was not significant. 
In a similar study, Henderson et al. (1982) used the GHQ and other psychiatric indices in a large community sample. Subjects were interviewed on 3 occasions 4 months apart. In one analysis of the data, a multiple regression of a number of social variables on the GHQ score was performed. Antecedent life stress accounted for only $3.4 \%$ of the variance in GHQ scores. In this analysis only subjects free of morbidity were included. The contribution of life events to psychological symptoms 4 months later, although statistically significant, was nonetheless trivial. In a second analysis (canonical analysis) the 4 psychiatric measures (including the Zung Depression Inventory and the GHQ) were collapsed into a single index of morbidity. In this analysis subjects with psychological morbidity at first interview were not excluded. This (in conjunction with a different index of psychological morbidity) may have accounted for a greater proportion of the variance $(8 \%$ versus $4 \%$ ) being explained by life events. In both analyses it is important to note that 'adversity' scores were, first, derived for the period before interview and not prior to the onset of symptoms and, secondly, included chronic difficulties which may not have been independent of pre-existing psychological morbidity. Despite the prospective design, the findings may not necessarily reflect a causal relation between life events and neurotic illness. It is of further interest to note that, unlike other prospective studies, personality variables were also included in their path analyses of the prospective data. The Neuroticism score on the Eysenck Personality Inventory (EPI) accounted for $69 \%$ of the variance in symptom scores.

This review has focused on prospective studies in which life event stress is measured prior to the onset of psychological morbidity. In such studies the likelihood that altered mood influences either the occurrence or the reporting of life events is largely excluded. These studies are to be distinguished from simple longitudinal studies in which events and illness are measured at the same point in time. Such studies are little better than cross-sectional designs (Paykel \& Tanner, 1976; Myers et al. 1972; Gersten et al. 1977; Tennant et al. 1981 b; Warheit, 1979). All but one of these studies (i.e. Murphy, 1983) share one limitation: they have used life event inventories which are far less sensitive than the approach used by Brown \& Harris (1978). While there are factors which might lead the use of these inventories to produce false negative results (namely that they lack sensitivity) this may be compensated by factors which lead to spuriously positive results; they do not, for instance, readily permit exclusion of all life events which might be caused by pre-existing psychological illness. Despite these limitations, the prospective findings overall do not seem to show that life events have any substantial causal role in neurotic illness.

The thrust of life stress research should continue with emphasis on prospective studies, using more sensitive measure of stress, with appropriate short follow-up periods, and finally should include some assessment of personality.

CHRISTOPHER TENNANT

\section{REFERENCES}

Andrews, G. (1981). A prospective study of life events and psychological symptoms. Psychological Medicine 11, 179-181.

Brown, G. W. \& Harris, T. (1978). The Social Origins of Depression. Tavistock: London.

Brown, G. W., Harris, T. O. \& Peto, J. (1973). Life events and psychiatric disorder. Part 2: Nature of the causal link. Psychological Medicine 3, 159-176.

Cassidy, W. L., Flanagan, N. B. \& Spellman, M. (1957). Clinical observations in manic-depressive disease. Journal of the American Medical Association 164, 1535-1546.

Cooper, B. \& Sylph, J. (1973). Life events and the onset of neurotic illness: an investigation in general practice. Psychological Medicine 3, $421-435$.

Eaton, W. W. (1978). Life events, social supports, and psychiatric symptoms: a re-analysis of the New Haven data. Journal of Health and Social Behaviour 19, 230-234.

Gersten, J. C., Langner, T. S., Eisenberg, J. G. \& Simcha-Fagan, O. (1977). An evaluation of the etiologic role of stressful life-change events in psychological disorders. Journal of Health and Social Behaviour 18, 228-244.

Grant, I., Yager, J., Sweetwood, H. L. \& Olshen, R. (1982). Life events and symptoms. Fourier analysis of time series from a three-year prospective inquiry. Archives of General Psychiatry 39, 598-605.

Hall, K. S., Dunner, D. L., Zeller, G. \& Fieve, R. R. (1977). Bipolar illness: a prospective study of life events. Comprehensive Psychiatry 18(5), 496-502.

Henderson, S., Byrne, D. G. \& Duncan-Jones, P. (1982). Neurosis and the Social Environment. Academic Press: London.

Holmes, T. H. \& Rahe, R. H. (1967). The social readjustment rating scale. Journal of Psychosomatic Research 11, 213-218.

Hornstra, R. K. \& Klassen, D. (1977). The course of depression. Comprehensive Psychiatry 18(2), 119-125.

Lloyd, C. (1980). Life events and depressive disorder reviewed. II. Events as precipitating factors. Archives of General Psychiatry 37, 541-548. 
Murphy, E. (1983). The prognosis of depression in old age. British Journal of Psychiatry 142, 111-119.

Myers, J. K., Lindenthal, J. J., Pepper, M. P. \& Ostrander, D. R. (1972). Life events and mental status: a longitudinal study. Journal of Health and Social Behaviour 13, 398-406.

Paykel, E. S. (1978). Contribution of life events to causation of psychiatric illness. Psychological Medicine 8, 245-253.

Paykel, E. S. \& Tanner, J. (1976). Life events, depressive relapse and maintenance treatment. Psychological Medicine 6, 481-485.

Tennant, C., Bebbington, P. \& Hurry, J. (1981a). The role of life events in depressive illness: is there a substantial causal relation? Psychological Medicine 11, 379-389.
Tennant, C., Bebbington, P. \& Hurry, J. (1981b). The short-term outcome of neurotic disorders in the community: the relation of remission to clinical factors and to 'neutralizing' life events. British Journal of Psychiatry 139, 213-220.

Theorell, T., Lind, E. \& Floderus, B. (1975). The relationship of disturbing life-changes and emotions to the early development of myocardial infarction and other serious illnesses. International Journal of Epidemiology 4(4), 281-293.

Warheit, G. (1979). Life events, coping, stress and depressive symptomatology. American Journal of Psychiatry 136(4B), 502-507. 